\title{
Superfluid density in gapless superconductor $\mathrm{CeCoIn}_{5}$
}

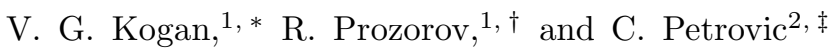 \\ ${ }^{1}$ Ames Laboratory and Department of Physics \& Astronomy, Iowa State University, Ames, Iowa 50011 \\ ${ }^{2}$ Department of Physics, Brookhaven National Laboratory, Upton, NY 11973
}

(Dated: 10 December 2007)

\begin{abstract}
Temperature dependence of the London penetration depth $\lambda$ measured in single crystals of $\mathrm{CeCoIn}_{5}$ is interpreted as caused by a strong pair-breaking scattering that makes the superconductivity in this compound gapless. For a gapless d-wave superconductor, we derive $\lambda=\lambda_{0} / \sqrt{1-\left(T / T_{c}\right)^{2}}$ caused by combined effect of magnetic and non-magnetic scattering in excellent agreement with the data in the full temperature range and with the gapless s-wave case of Abrikosov and Gor'kov. We also obtain the slope of the upper critical field at $T_{c}$ that compares well with the measured slope.
\end{abstract}

PACS numbers: 74.20.-z, 74.20.Rp, 74.70.Tx

The heavy-fermion superconductor $\mathrm{CeCoIn}_{5}$ is still under intensive scrutiny after its discovery in 2001 [1]. This is clean (the mean-free path exceeds by much the coherence length $\xi_{a b} \approx 80 \AA$ ), nearly two-dimensional (with small 3D pockets) [2], d-wave superconductor [3]. In the normal phase for $T>T_{c}=2.3 \mathrm{~K}$, this material is a strong paramagnet [5]. The main interest of the community has been focussed on low temperatures and high fields where the inhomogeneous Fulde-FerrellLarkin-Ovchinnikov (FFLO) phase is suspected to exist. In this work we are interested in zero-field superfluid density and the $c$ directed upper critical field $H_{c 2}$ near $T_{c}$, the domain removed from complications of FFLO and paramagnetic constrains. Understanding the ground state properties is of utmost importance for definite predictions about existence of more complex phases such as FFLO.

Single crystals of $\mathrm{CeCoIn}_{5}$ were grown from an In flux by combining stoichiometric amounts of $\mathrm{Ce}$ and Co with excess In in an alumina crucible and encapsulating the crucible under vacuum in a quartz tube. Details of the crystal growth are described elsewhere [1]. The crystals used were $1 \times 1 \times 0.2 \mathrm{~mm}^{3}$ and magnetic measurements showed practically no hysteresis.

The magnetic penetration depth was measured with a tunnel-diode resonator sensitive to changes in $\lambda$ of about $1 \AA$. Details of the technique are described elsewhere [12]. In short, properly biased tunnel diode maintains self-resonating tank circuit on its resonant frequency $\omega \sim 14 \mathrm{MHz}$. A sample is inserted into the coil on a sapphire rod. The changes in the effective inductance cause a shift in $\omega$ proportional to the real part of the dynamic magnetic susceptibility. The system is calibrated by matching the $T$ dependent skin depth just above $T_{c}$. To probe $\lambda_{a b}$, a small ac magnetic field $(\sim 20 \mathrm{mOe})$ is applied along the c-axis, so that the screening currents are flowing in the $a b$ plane [12].

The London penetration depth $\lambda_{a b}(T)$ as a function of temperature shown in Fig.1]. With excellent accuracy,

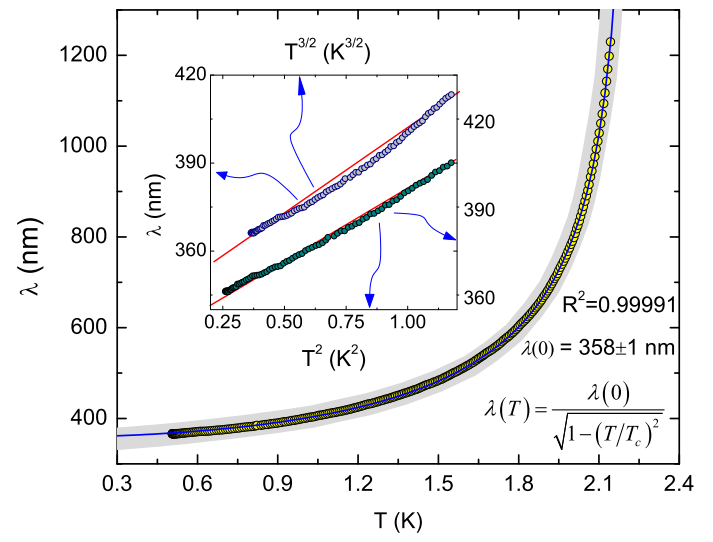

FIG. 1: (Color online) The data on $\lambda(T)$ and the fit to Eq. (1) with $\lambda(0)$ as the single fitting parameter. Grey line: data from Ref. [6]. Inset: low temperature part of $\lambda(T)$ replotted vs. $T^{3 / 2}$ and $T^{2}$ for comparison.

the data from $0.5 \mathrm{~K}$ all the way to $2.1 \mathrm{~K}$ are described by

$$
\lambda_{a b}=\lambda(0) / \sqrt{1-t^{2}}, \quad t=T / T_{c} .
$$

In fact, our data are close to those reported in Ref. [6] (the grey band in Fig.11); our interpretation, however, is quite different.

We note that the superfluid density $1 / \lambda^{2}$ deviates from the zero- $T$ value approximately as $T^{2}$ at low $T$ 's, unlike both the s-wave exponentially weak behavior and the clean d-wave linear $T$ dependence. On the other hand, we recall that Abrikosov and Gor'kov (AG) in the seminal paper [7] on pair-breaking by magnetic impurities in isotropic s-wave materials found the dependence (1) in the whole temperature range from 0 to $T_{c}$ for a strong spin-flip scattering when $T_{c}$ is suppressed to nearly zero and the superconductivity is gapless.

This suggests that a similar situation takes place in $\mathrm{CeCoIn}_{5}$, although the reason for the pair-breaking may not be the spin-flip scattering on independent magnetic impurities of AG. Instead, it might be scattering on ex- 
citations of the Kondo system of interacting local moments [8]. The situation is far from being transparent as is shown by transport measurements with magnetic and non-magnetic substitutions [9]. Alternatively, the gaplessness might be caused by only a part of the Fermi surface being fully gapped in multiband scenarios [10, 11].

Since our technique only provides information on the change $\Delta \lambda(T)$, we compare our data with those of Ref. [6] where $\lambda(0)$ was estimated from the surface impedance data. By shifting our $\Delta \lambda(T)$ (circles in Fig. (1) we obtain one-to-one correspondence with the data of Ref. [6] shown in Fig. 1 by a grey band (the width is artificial - otherwise the data just collapse on top of each other). The solid line is the fit to Eq. (11) in the full temperature interval. The only fitting parameter, $\lambda(0)=358 \mathrm{~nm}$, is larger than $281 \mathrm{~nm}$ obtained in Ref. [6] from the low $T$ part of the data (shown in the inset). The fit is of a high precision reflected in the value of the "coefficient of determination" $R^{2}=0.99991$ (for the perfect fit $R^{2}=1$ ).

Inset to Fig.1 shows comparison of the data in the low-temperature domain with the form suggested in [6], $\Delta \lambda=\lambda-\lambda(0) \propto T^{3 / 2}$, and with the $\Delta \lambda \propto T^{2}$, expected from our model (displaced vertically by $20 \mathrm{~nm}$ for clarity). Straight lines show clearly that the $T^{2}$ dependence describes the data better.

Long experience of dealing with pair-breaking effects reveals that all of them are described by the AG formalism, provided the dimensionless pair-breaking parameters are properly defined for each particular case [13]. We therefore choose the AG scheme and characterize the scattering by two parameters

$$
\rho=\hbar / 2 \pi T_{c} \tau, \quad \rho_{m}=\hbar / 2 \pi T_{c} \tau_{m}
$$

where $T_{c}$ is the critical temperature (not to confuse with hypothetical $T_{c 0}$ of the material free of scattering), $1 / \tau$ and $1 / \tau_{m}$ are the transport and the pair-breaking scattering rates. As mentioned, AG find $\lambda^{-2} \propto 1-t^{2}$ for sufficiently strong pair-breaking in dirty isotropic s-wave materials with $\rho \gg \rho_{m}$. The material of interest here is a clean d-wave [3]; we show below that this $T$ dependence holds in this situation as well.

Within microscopic theory, the penetration of weak magnetic fields into superconductors is evaluated by first solving for the unperturbed zero-field state and then treating effects of small fields as perturbations. Perhaps, the simplest for this task is the Eilenberger quasiclassical approach [14]. Formally, it consists of equations for Eilenberger functions $f(\boldsymbol{r}, \boldsymbol{v}, \omega), f^{+}$, and $g$ which originate from Gor'kov's Green's functions integrated over the energy near the Fermi surface to exclude fast spatial oscillations on the scale $1 / k_{F}$ :

$$
\begin{aligned}
\boldsymbol{v} \boldsymbol{\Pi} f & =\frac{2 \Delta g}{\hbar}-2 \omega f+\frac{g}{\tau^{-}}\langle f\rangle-\frac{f}{\tau^{+}}\langle g\rangle, \\
-\boldsymbol{v} \boldsymbol{\Pi}^{*} f^{+} & =\frac{2 \Delta^{*} g}{\hbar}-2 \omega f^{+}+\frac{g}{\tau^{-}}\left\langle f^{+}\right\rangle-\frac{f^{+}}{\tau^{+}}\langle g\rangle, \\
1 & =g^{2}+f f^{+} .
\end{aligned}
$$

Here, $\boldsymbol{v}$ is the Fermi velocity, $\boldsymbol{\Pi}=\nabla+2 \pi i \boldsymbol{A} / \phi_{0}, \phi_{0}$ is the flux quantum. $\Delta$ is the order parameter which may depend on the position $\boldsymbol{k}_{F}$ at the Fermi surface (or on $\boldsymbol{v}$ ) in cases other than the isotropic s-wave; for a d-wave material with a simple cylindrical Fermi surface the order parameter can be written as

$$
\Delta=\Psi(\boldsymbol{r}, T) \Omega, \quad \Omega=\sqrt{2} \cos 2 \varphi,
$$

where $\varphi$ is the azimuthal angle on the Fermi cylinder and $\Omega$ is normalized to have $\left\langle\Omega^{2}\right\rangle=1$.

Further, $\omega$ are Matsubara frequencies defined by $\hbar \omega=$ $\pi T(2 n+1)$ with an integer $n,\langle\ldots\rangle$ denote averages over the Fermi surface, and

$$
\frac{1}{\tau^{ \pm}}=\frac{1}{\tau} \pm \frac{1}{\tau_{m}} .
$$

The system (3)-(5) should be complemented with the self-consistency equation for the order parameter and with an expression for the current density. For the dwave symmetry, both magnetic and non-magnetic scattering suppress the critical temperature [15]. The selfconsistency equation in the form taking this into account, reads:

$$
\ln \frac{T_{c}}{T}=\sum_{n=0}^{\infty}\left(\frac{1}{n+1 / 2+\rho^{+} / 2}-\frac{2 \pi T}{\Psi}\langle\Omega f\rangle\right),
$$

where $\rho^{+}=\rho+\rho_{m}$. Finally, the current density expression completes the Eilenberger system:

$$
\boldsymbol{j}=-4 \pi|e| N(0) T \operatorname{Im} \sum_{\omega>0}\langle\boldsymbol{v} g\rangle
$$

$N(0)$ is the total density of states at the Fermi level per one spin.

Calculation of $\lambda\left(T ; \tau, \tau_{m}\right)$ for arbitrary $\tau$ 's is difficult analytically. However, for a strong $T_{c}$ suppression, the problem is manageable. We begin with uniform zerofield state for which $\rho+\rho_{m}$ is close to the critical value where $T_{c} \rightarrow 0$; in this state $f \ll 1$ and $g \approx 1-f^{2} / 2$ in the whole temperature range [7]. One can look for solutions of Eq. (3) as $f=f_{1}+f_{2}$ with $f_{2} \ll f_{1}$. In the lowest approximation Eq. (3) yields:

$$
2 \Delta / \hbar-2 \omega f_{1}+\left\langle f_{1}\right\rangle / \tau^{-}-f_{1} / \tau^{+}=0 .
$$

Since $\langle\Delta\rangle=0$ for the d-wave materials, averaging of this equation over the Fermi surface gives $\left\langle f_{1}\right\rangle=0$ as well. Taking this into account we have

$$
f_{1}=\Delta / \hbar \omega^{+}, \quad \omega^{+}=\omega+1 / 2 \tau^{+} .
$$


The next approximation yields:

$$
f_{2}=\frac{\Delta}{2 \hbar^{3} \omega^{+3}}\left(\frac{\left\langle\Delta^{2}\right\rangle}{2 \tau^{+} \omega^{+}}-\Delta^{2}\right)
$$

Making use of $\Delta=\Omega \Psi,\left\langle\Omega^{2}\right\rangle=1,\left\langle\Omega^{4}\right\rangle=3 / 4$ for a Fermi cylinder, we evaluate:

$$
\left\langle\Omega\left(f_{1}+f_{2}\right)\right\rangle=\frac{\Psi}{\hbar \omega^{+}}-\frac{\Psi^{3}\left(1-6 \omega \tau^{+}\right)}{16 \hbar^{3} \omega^{+4} \tau^{+}} .
$$

Substitute this in Eq. (8), express the sums in terms of digamma functions, and utilize the asymptotic expansion $\psi(z+1 / 2) \approx \ln z+1 / 24 z^{2}$ for $z=\rho^{+} / 2 t \gg 1$ :

$$
\begin{aligned}
& -\ln t=\psi\left(\frac{\rho^{+}}{2 t}+\frac{1}{2}\right)-\psi\left(\frac{\rho^{+}}{2}+\frac{1}{2}\right) \\
& -\frac{3 \Psi^{2}}{32 \pi^{2} T^{2}}\left[\psi^{\prime \prime}\left(\frac{\rho^{+}}{2 t}+\frac{1}{2}\right)-\frac{\rho^{+}}{9 t} \psi^{\prime \prime \prime}\left(\frac{\rho^{+}}{2 t}+\frac{1}{2}\right)\right] \\
& \approx-\ln t+\frac{t^{2}-1}{6 \rho^{+2}}+\frac{\Psi^{2}}{48 \pi^{2} T_{c}^{2} \rho^{+2}} .
\end{aligned}
$$

Hence, we obtain:

$$
\Psi^{2}=8 \pi^{2}\left(T_{c}^{2}-T^{2}\right) .
$$

This differs from the result for isotropic s-wave superconductors with magnetic impurities of nearly critical concentration, by a 4 times larger pre-factor [7]. The ratio

$$
\Delta_{\max }(0) / T_{c}=4 \pi
$$

is considerably larger than 1.764 of the BCS theory for isotropic s-wave materials and 2.14 for the clean isotropic $\mathrm{d}$-wave case. This ratio estimated from the tunneling data on $\mathrm{CeCoIn}_{5}$ is about 10 [16].

It is worth noting that even without magnetic scatterers, the superconductivity in d-wave materials becomes gapless in the domain of interest here with $\rho^{+} \gg 1$. To see this, examine the density of states $N(\epsilon)=N(0) \operatorname{Re} g(\hbar \omega \rightarrow i \epsilon)$ using $g=1-f^{2} / 2=$ $1-\left(\Delta / \hbar \omega^{+}\right)^{2} / 2$ :

$$
\frac{N(\epsilon)}{N(0)}=1-\frac{2 \Delta^{2} \tau^{+2}}{\hbar^{2}} \frac{1-\varepsilon^{2}}{\left(1+\varepsilon^{2}\right)^{2}}, \quad \varepsilon=\frac{2 \tau^{+} \epsilon}{\hbar} .
$$

Hence, at zero energy, $N(\epsilon)$ has a non-zero minimum (i.e., the superconductivity is gapless), whereas the maximum of $N(\epsilon)$ is reached at $\epsilon_{m}=\hbar \sqrt{3} / 2 \tau^{+}$.

Weak supercurrents and fields leave the order parameter modulus unchanged, but cause the condensate to acquire an overall phase $\theta(\boldsymbol{r})$. We therefore look for perturbed solutions in the form:

$$
\begin{gathered}
\Delta=\Delta_{0} e^{i \theta}, \quad f=\left(f_{0}+f_{1}\right) e^{i \theta}, \\
f^{+}=\left(f_{0}+f_{1}^{+}\right) e^{-i \theta}, \quad g=g_{0}+g_{1},
\end{gathered}
$$

where the subscript 1 denotes small corrections. In the London limit, the only coordinate dependence is that of the phase $\theta$, i.e., $f_{1}, g_{1}$ can be taken as $\boldsymbol{r}$ independent.
The Eilenberger equations (3)-(5) provide the corrections among which we need only $g_{1}$ :

$$
g_{1}=\frac{i \hbar f_{0}^{2} \boldsymbol{v} \boldsymbol{P}}{2\left(\tilde{\Delta} f_{0}+\hbar \tilde{\omega} g_{0}\right)} \approx \frac{i f_{0}^{2} \boldsymbol{v} \boldsymbol{P}}{2 \omega^{+}} .
$$

Here $\boldsymbol{P}=\nabla \theta+2 \pi \boldsymbol{A} / \phi_{0} \equiv 2 \pi \boldsymbol{a} / \phi_{0}$ with the "gauge invariant vector potential" $\boldsymbol{a}$, and

$$
\tilde{\Delta}=\Delta+\hbar\langle f\rangle / 2 \tau^{-}, \quad \tilde{\omega}=\omega+\langle g\rangle / 2 \tau^{+} .
$$

In the case of interest, $f_{0} \approx \Delta / \hbar \omega^{+} \ll 1$, and the denominator in Eq. (19) is taken in the lowest order. We now substitute $g_{0}+g_{1}$ in the current (9) and compare the result with $4 \pi j_{i} / c=-\left(\lambda^{2}\right)_{i k}^{-1} a_{k}$ to obtain:

$$
\lambda_{a a}^{-2}=\frac{32 \pi e^{2} N(0) v^{2}}{c^{2} \rho^{+2}}\left(1-t^{2}\right) .
$$

Using the data of Fig. 11 with estimates for $N(0)$ and $v$ taken from [5], we obtain $\rho^{+} \approx 8$. Estimates of [3] yield $\rho^{+} \approx 5$. Hence, the statement that $\mathrm{CeCoIn}_{5}$ is gapless is not only in excellent agreement with the $T$ dependence of $\lambda$, but the scattering parameter $\rho^{+}$is sufficiently large which is needed for our model to hold.

There are quite a few reports of $H_{c 2}(T)$ for $\mathrm{CeCoIn}_{5}$, see, e.g., [17]. Our data are shown in Fig.2. For a strong pair-breaking model with fixed scattering parameters $H_{c 2} \propto\left(1-t^{2}\right)$ [7] that is clearly different from the experimental behavior. In a strong paramagnet such as $\mathrm{CeCoIn}_{5}$, the magnetic scattering rate (and $\rho_{m}$ ) may itself depend on the applied field, making our model with a constant $\rho_{m}$ inapplicable per se along the whole $H_{c 2}(T)$ curve. However, near $T_{c}$ where $H_{c 2} \rightarrow 0$, we expect the model to hold. We, therefore, evaluate only $H_{c 2}(T)$ near $T_{c}$; in other words, we derive the Ginzburg-Landau equation containing the coherence length $\xi$ for the gapless case with a strong pair-breaking.

Near $T_{c}$, we look for a solution $f$ of Eq. (3) as an expansion in two small parameters

$$
\frac{\Delta}{\hbar \omega^{+}} \sim \frac{\Delta}{T_{c}} \sim \delta t^{1 / 2}, \text { and } \frac{v \boldsymbol{\Pi} \Delta}{\hbar \omega^{+2}} \sim \frac{\Delta}{T_{c} \xi} \sim \delta t
$$

where $\delta t=1-T / T_{c}$; smallness of the second parameter comes from the "slow variation" requirement. We obtain after simple algebra:

$$
f=\frac{\Delta}{\hbar \omega^{+}}-\frac{\boldsymbol{v} \Pi \Delta}{2 \hbar \omega^{+2}}+\mathcal{O}\left(\delta t^{3 / 2}\right) .
$$

Further, the self-consistency Eq. (8) which now reads as

$$
\frac{\Psi}{2 \pi T_{c}} \delta t=\sum_{\omega>0}\left(\frac{\Psi}{\hbar \omega+\pi T \rho^{+}}-\langle\Omega f\rangle\right),
$$

should be taken into account. To evaluate $\langle\Omega f\rangle$, we substitute $g=1-f f^{+} / 2$ in Eq. (3), multiply it by $\Omega / \omega^{+}$, 
and take average over the Fermi surface:

$$
\begin{aligned}
\frac{1}{2 \omega^{+}}\langle\Omega v \Pi f\rangle & =\frac{\Psi}{\hbar \omega^{+}}-\langle\Omega f\rangle \\
& -\frac{3 \Psi|\Psi|^{2}}{8 \hbar^{3} \omega^{+3}}+\frac{\Psi|\Psi|^{2}}{4 \hbar^{3} \omega^{+4} \tau^{+}} .
\end{aligned}
$$

Writing the last two terms, we can take $f$ in the lowest order; also we make use of $\Delta=\Psi \Omega,\left\langle\Omega^{2}\right\rangle=1$, and of $\left\langle\Omega^{4}\right\rangle=3 / 4$. On the left-hand side we have:

$$
\begin{array}{r}
\frac{1}{2 \hbar \omega^{+2}}\left\langle\Omega^{2} \boldsymbol{v} \boldsymbol{\Pi} \Psi\right\rangle+\frac{1}{4 \hbar \omega^{+3}}\left\langle\Omega^{2}(\boldsymbol{v} \boldsymbol{\Pi})^{2} \Psi\right\rangle \\
=\frac{1}{4 \hbar \omega^{+3}}\left\langle\Omega^{2} v_{i} v_{k}\right\rangle \Pi_{i} \Pi_{k} \Psi
\end{array}
$$

summation over the repeated indices is implied.

We now sum up Eq. (24) over $\omega$ and take $\sum\langle\Omega f\rangle$ from the self-consistency Eq. (23). All sums obtained are expressed in terms of di-gamma functions, for which the large argument asymptotics can be used. We are interested in the field along the $c$ crystal axis, whereas the plane $a b$ can be taken as isotropic. After a straightforward algebra one obtains the GL equation in the standard form

$$
-\xi^{2} \Pi^{2} \Psi=\Psi\left(1-|\Psi|^{2} / \Psi_{0}^{2}\right)
$$

with

$$
\xi^{2}=\frac{3 \hbar^{2} v^{2}}{32 \pi^{2} T_{c}^{2} \delta t}, \quad \Psi_{0}^{2}=16 \pi^{2} T_{c}^{2} \delta t .
$$

We note that $\Psi_{0}^{2}$ is the zero-field order parameter of Eq. (15) obtained there by a different method.

Although the scattering parameters do not enter explicitly the coherence length, they affect $\xi$ through $T_{c}\left(\rho^{+}\right)$. Equation (27) shows that the scattering, whatever it is, enhances $\xi$. In other words, in the gapless dwave regime, the effect of scattering upon the coherence length is opposite to the familiar s-wave situation where $\xi$ is suppressed by scattering. When the scattering approaches the critical value for which $T_{c} \rightarrow 0, \xi$ diverges. The slope of the upper critical field at $T \rightarrow T_{c}$ follows:

$$
H_{c 2}^{\prime}\left(T_{c}\right)=-\frac{16 \pi \phi_{0} T_{c} k_{B}^{2}}{3 \hbar^{2} v^{2}},
$$

where the temperature is given in Kelvins. Hence, the slope decreases when scattering is intensified. This unusual behavior is confirmed by the data on $H_{c 2}$ for low concentration La doping of $\mathrm{Ce}_{1-x} \mathrm{La}_{x} \mathrm{CoIn}_{5}$ [5]. Moreover, we note that the Fermi velocity can now be expressed in terms of the measured slope and $T_{c}$. This yields $v=1.1 \times 10^{6} \mathrm{~cm} / \mathrm{s}$, the value between $v=2 \times$ $10^{6} \mathrm{~cm} / \mathrm{s}$ obtained using the heat capacity and resistivity data in Ref. [5] and $v=0.77 \times 10^{6} \mathrm{~cm} / \mathrm{s}$ as estimated in Ref. [3].

To conclude, we show that the data on the $T$ dependence of the penetration depth in the full temperature

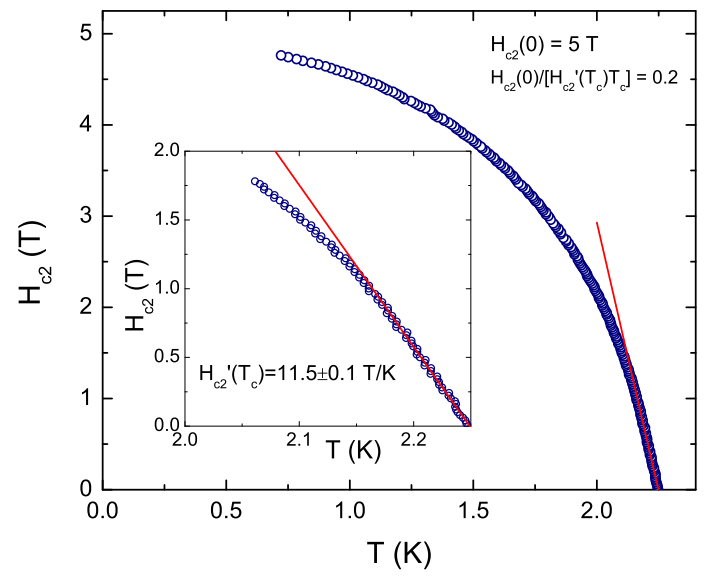

FIG. 2: (Color online) The upper critical field $H_{c 2}(T) \| \boldsymbol{c}$. Inset shows the domain near $T_{c}$ with an estimate of the slope.

range and on the upper critical field near $T_{c}$ strongly support the notion that the superconductivity in $\mathrm{CeCoIn}_{5}$ is gapless.

We thank Paul Canfield and Sergey Bud'ko for many useful discussions. The work at the Ames Laboratory is supported by the Office of Basic Energy Sciences of the US Department of Energy under the Contract No. DEAC02-07CH11358. R.P. acknowledges support of Alfred P. Sloan Foundation.

* Electronic address: kogan@ameslab.gov

† Electronic address: prozorov@ameslab.gov

‡ Electronic address: petrovic@bnl.gov

[1] C. Petrovic et al, J. Phys.: Condens. Matter 13, L337 (2001).

[2] R. Settai et al , J. Phys. Cond. Matt. 13, L627 (2001); R. Settai, T. Takeuchi, and Y. Onuki, J. Phys. Soc. Jpn, 76, 051003 (2007).

[3] R. Movshovich et al, Phys. Rev. Lett. , 86, 5152 (2001).

[4] K. Izawa et al, Phys. Rev. Lett. 87, 057002 (2001).

[5] C. Petrovic et al, Phys. Rev. B , 66, 054534 (2002).

[6] S. Ozcan et al, Europhys. Lett. 62, 412 (2003).

[7] A. A. Abrikosov and L. P. Gor'kov, Zh. Eksp. Teor. Fiz. 39, 1781 (1060) [Sov. Phys. JETP, 12, 1243 (1961)].

[8] S. Nakatsuji, D. Pines, and Z. Fisk, Phys. Rev. Lett. 92, 016401 (2004).

[9] J. Piglione et al, cond-mat/0705.2592.

[10] M. A. Tanatar et al, Phys. Rev. Lett., 95, 06702 (2005).

[11] V. Barzykin and L. P. Gor'kov, Phys. Rev. B 76, 014509 (2007).

[12] R. Prozorov et al, Phys. Rev. B 62, 115 (2000); R. Prozorov and R. W. Giannetta, Superc. Sci. Techn. (topical review) 19, R41 (2006).

[13] K. Maki in Superconductivity ed by R. D. Parks, Marcel Dekker, New York, 1969, v.2, p.1035.

[14] G. Eilenberger, Z. Phys. 214, 195 (1968).

[15] L. A. Openov, Pis'ma Zh. Eksp. Teor, Fiz. 66, 627 (1997). 
[16] W. K. Park et al, cond-mat/0706.1376.

[17] A. Bianchi et al, Phys. Rev. Lett. 91, 187004 (2003). 